\title{
TRANSFORMATION DE FOURIER-SATO ET OPÉRATEURS PSEUDO-DIFFÉRENTIELS NON-LOCAUX
}

\author{
Ryuichi ISHIMURA \\ (Received 15 November 2005)
}

\begin{abstract}
In this paper, first, we re-establish the notion of non-local pseudodifferential operators introduced by the author, more intrinsically by means of the Fourier-Sato transformation of the sheaf of holomorphic forms. By using this, we propose the construction of the composition of non-local pseudo-differential operators functorially.
\end{abstract}

\section{Introduction}

Dans l'article précédent [1], nous avons introduit la notion d'un opérateur pseudodifférentiel non-local et établi leurs compositions au langage du cohomologie. Cette classe des opérateurs contient les opérateurs pseudo-différentiels, les opérateurs de convolution et aussi les opérateurs différentiels-différences à coefficient variable.

Dans present article, nous proposons de rétablir la notion d'un opérateur pseudo-différentiel non-local, plus intrinsèquement, au moyen de transformation de Fourier-Sato, développé dans [3], du faisceau des formes holomorphes. Cela nous permet de reconstruire, fonctoriellement, la composition des deux tels opérateurs.

\section{La transformation de Fourier-Sato}

Dans présent article, nous emploierons les notations du livre de Kashiwara et Schapira [2], que nous appellerons le livre jaune. Nous commençons par nous rappeller quelques notations de la section 3.7 du livre jaune et les propositions qui

2000 Mathematics Subject Classification: Primary 32W25, 35R50.

Keywords and Phrases: pseudo-differential operators; partial differential equations of infinite order; non-local operators. 
se démontrent comme les Propositions similaires du même livre. Soient d'abord, $Z$ un espace localement compact, $\tau: E \rightarrow Z$ un fibré vectoriel réel au fibre de dimension $n$ et $\pi: E^{*} \rightarrow Z$ son dual. Désignos par $E \times E_{Z}^{*}$ le produit-fibre de $E$ et $E^{*}$, i.e. $E \times E^{*}:=\left\{(w, \zeta) \in E \times E^{*} \mid \tau(w)=\pi(\zeta)\right\}$, et $E \stackrel{\rho_{1}}{\longleftarrow} E \times E_{Z}^{*} \stackrel{\rho_{2}}{\longrightarrow} E^{*}$ les projections :

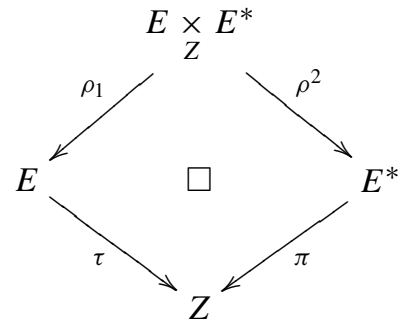

Prenons une forme continue $L: E \underset{Z}{\times} E^{*} \rightarrow \mathbb{R}$ bilnéaire en chaque fibre et une fonction continue $a: E^{*} \rightarrow \mathbb{R}$ homogène en chaque fibre, et définissons

$$
P:=\left\{(w, \zeta) \in \underset{Z}{E \underset{Z}{\times}} E^{*} \mid L(w, \zeta) \geqslant a(\zeta)\right\}
$$

On désigne par $D^{+}(E)$ et $D^{+}\left(E^{*}\right)$, les catégories dérivées des complexes des faiceaux du $\mathbb{C}$-module sur, respectivement, $E$ et $E^{*}$, bornés à droite.

Définition 2.1. Pour tout objet $F \in D^{+}(E)$, on pose

$$
\mathcal{F}^{P} F:=R \rho_{2 *} \circ R \Gamma_{P} \circ \rho_{1}^{-1} F \in D^{+}\left(E^{*}\right)
$$

et on l'appelle la transformation de Fourier-Sato de $F$ portée par $P$.

Paralèllement à la proposition 3.7.12 (ii) de [2], on a la proposition suivante :

Proposition 2.2. Pour tout $F \in D^{+}(E)$ et tout ouvert $U \subset E^{*}$, on a

$$
R \Gamma\left(U ; \mathcal{F}^{P} F\right) \simeq R \Gamma_{U^{P}}\left(\tau^{-1} \pi(U) ; F\right)
$$

où l'on a posé

$$
\begin{aligned}
U^{P} & :=\left\{w \in \tau^{-1} \pi(U) \mid(w, \zeta) \in P(\forall \zeta \in U)\right\} \\
& =\left\{w \in \tau^{-1} \pi(U) \mid L(w, \zeta) \geqslant a(\zeta)(\forall \zeta \in U)\right\} .
\end{aligned}
$$

Ensuite, soient $Z_{1}, Z_{2}$ deux espaces localement compacts et $\tau_{1}: E_{1} \rightarrow Z_{1}, \tau_{2}$ : $E_{2} \rightarrow Z_{2}$ deux fibrés vectoriels, $\pi_{1}: E_{1}^{*} \rightarrow Z_{1}, \pi_{2}: E_{2}^{*} \rightarrow Z_{2}$ leurs duals et 
$E_{i} \stackrel{\rho_{1 i}}{\longleftarrow} E_{i} \underset{Z_{i}}{\times} E_{i}^{*} \stackrel{\rho_{2 i}}{\longrightarrow} E_{i}^{*}$ les projections pour $i=1,2$. On suppose que les ensembles

$$
\begin{aligned}
& P_{1}=\left\{(w, \zeta) \in E \underset{Z}{\times} E^{*} \mid L_{1}(w, \zeta) \geqslant a_{1}(\xi)\right\}, \\
& P_{2}=\left\{(w, \zeta) \in \underset{Z}{E \times} E^{*} \mid L_{2}(w, \zeta) \geqslant a_{2}(\zeta)\right\}
\end{aligned}
$$

soient, comme (2.1), définis, $L_{1}, L_{2}, a_{1}, a_{2}$ étant les fonctions correspondants. On a le diagramme :

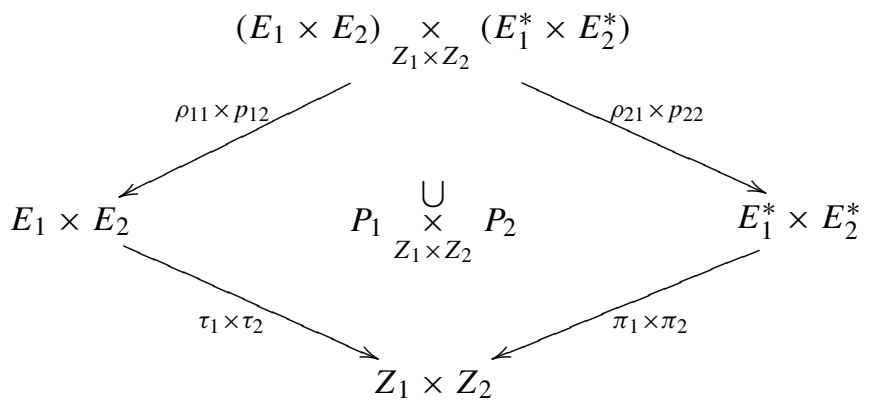

Soient $E_{1} \stackrel{q_{1}}{\longleftarrow} E_{1} \times E_{2} \stackrel{q_{2}}{\longrightarrow} E_{2}$ ou $E_{1}^{*} \stackrel{p_{1}}{\longleftarrow} E_{1}^{*} \times E_{2}^{*} \stackrel{p_{2}}{\longrightarrow} E_{2}^{*}$ les projections. Pour tous $F_{1} \in D^{+}\left(E_{1}\right)$ et $F_{2} \in D^{+}\left(E_{2}\right)$ ou tous $G_{1} \in D^{+}\left(E_{1}^{*}\right)$ et $G_{2} \in D^{+}\left(E_{2}^{*}\right)$, on définit le produit tensoriel extérieur :

$$
\begin{aligned}
\stackrel{\mathbb{L}}{\otimes} F_{2} & :=q_{1}^{-1} F_{1} \stackrel{\mathbb{L}}{\otimes} q_{2}^{-1} F_{2} \in D^{+}\left(E_{1} \times E_{2}\right), \\
G_{1} & \stackrel{\mathbb{L}}{\otimes} G_{2}:=p_{1}^{-1} G_{1} \stackrel{\mathbb{L}}{\otimes} p_{2}^{-1} G_{2} \in D^{+}\left(E_{1}^{*} \times E_{2}^{*}\right)
\end{aligned}
$$

(voir [2, 2.3.26]). Posons $P:=P_{1} \underset{Z_{1} \times Z_{2}}{\times} P_{2}$. Alors on a évidement :

Proposition 2.3. Pour tous $F_{1} \in D^{+}\left(E_{1}\right)$ et $F_{2} \in D^{+}\left(E_{2}\right)$, on a $\mathcal{F}^{P}\left(F_{1} \stackrel{\mathbb{L}}{\square} F_{2}\right) \in$ $D^{+}\left(E_{1}^{*} \times E_{2}^{*}\right)$ et on a l'isomorphisme

$$
\mathcal{F}^{P_{1}} F_{1} \stackrel{\mathbb{L}}{\otimes} \mathcal{F}^{P_{2}} F_{2} \stackrel{\sim}{\rightarrow} \mathcal{F}^{P}\left(F_{1} \stackrel{\mathbb{L}}{\otimes} F_{2}\right)
$$

En suite, faisons un changement de base: soit $f: Z^{\prime} \rightarrow Z$ un morphisme d'espace localement compact et soit $E \stackrel{\tau}{\rightarrow} Z$ un fibré vectoriel. Posons $E^{\prime}:=Z_{Z}^{\prime} \underset{Z}{E} E$ et considérons le changement de base $f_{\tau}: E^{\prime} \rightarrow E$. En écrivant $\tau^{\prime}: E^{\prime} \rightarrow Z^{\prime}$ 
la projection, on a :

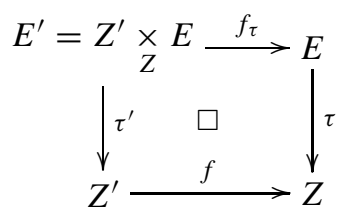

et par dualité, en posant $E^{\prime *}:=Z_{Z}^{\prime} \underset{Z}{\times} E^{*}$ et en désignant $\pi: E^{*} \rightarrow Z$ et $\pi^{\prime}: E^{\prime *} \rightarrow Z^{\prime}$ les projections, on a :

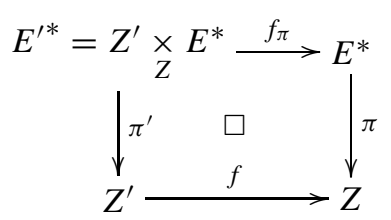

Pour $P \subset E \underset{Z}{\times} E^{*}$ défini par (2.1) avec $L, a$, on pose $P^{\prime}:=Z_{Z}^{\prime} \underset{Z}{\times} P$. Alors par définition, on a les propositions suivantes :

Proposition 2.4. Pour tout $F \in D^{+}(E)$, on a des ishomorphismes

$$
\begin{aligned}
\mathcal{F}^{P^{\prime}}\left(f_{\tau}^{!} F\right) & \simeq f_{\pi}^{!} \mathcal{F}^{P} F, \\
\mathcal{F}^{P^{\prime}}\left(f_{\tau}^{-1} F\right) & \simeq f_{\pi}^{-1} \mathcal{F}^{P} F .
\end{aligned}
$$

Pour tout $G \in D^{+}\left(E^{\prime}\right)$, on a des ishomorphismes

$$
\begin{aligned}
\mathcal{F}^{P}\left(R f_{\tau *} G\right) & \simeq R f_{\pi *}\left(\mathcal{F}^{P^{\prime}} G\right), \\
\mathcal{F}^{P}\left(R f_{\tau !} G\right) & \simeq R f_{\pi !}\left(\mathcal{F}^{P^{\prime}} G\right) .
\end{aligned}
$$

En suite, soient $E_{1} \stackrel{\tau_{1}}{\rightarrow} Z$ et $E_{2} \stackrel{\tau_{2}}{\rightarrow} Z$ deux fibrés vectoriels au-dessus de $Z$ et soit $f: E_{1} \rightarrow E_{2}$ un morphisme du fibré vectoriel. Désignons $\operatorname{par}^{t} f: E_{2}^{*} \rightarrow E_{1}^{*}$ le dual de $f$. Prenons $P=\{(w, \zeta) \mid L(w, \zeta) \geqslant a(\zeta)\} \subset E_{1} \underset{Z}{\times} E_{1}^{*}$ comme dans (2.1) et considérons le diagramme

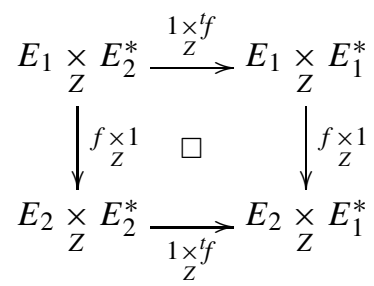


On a la proposition suivante :

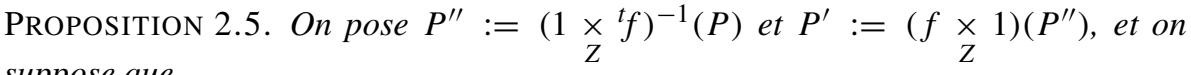
suppose que

$$
P^{\prime \prime}=\left(\underset{Z}{\underset{Z}{\times} 1)^{-1}\left(P^{\prime}\right)}\right. \text {. }
$$

Alors pour tout $F \in D^{+}\left(E_{1}\right)$, on a des isomorphismes

$$
\begin{aligned}
&{ }^{t} f^{-1}\left(\mathcal{F}^{P} F\right) \simeq \mathcal{F}^{P^{\prime}} R f_{*} F, \\
&{ }^{t} f^{!}\left(\mathcal{F}^{P} F\right) \stackrel{\mathbb{L}}{\otimes} \omega_{E_{2}^{*} / Z} \simeq \mathcal{F}^{P^{\prime}} R f_{*} F
\end{aligned}
$$

où $\omega_{E_{2}^{*} / Z}:=\tau_{2}^{!} \mathbb{C}_{Z}$

On a aussi, en écrivant $\omega_{E_{2}^{*} / E_{1}^{*}}:={ }^{t} f^{!} \mathbb{C}_{E_{1}^{*}}$ :

Proposition 2.6. On pose $\tilde{P}:=(\underset{Z}{f \underset{Z}{\times}})(P)$ et et on suppose que

$$
P=(f \underset{Z}{\times} \underset{Z}{ } 1)^{-1}(\tilde{P}) .
$$

Alors pour $G \in D^{+}\left(E_{2}\right)$, on a le morphisme canonique

$$
R^{t} f_{!}\left(\mathcal{F}^{P^{\prime}} G\right) \stackrel{\mathbb{L}}{\otimes} \omega_{E_{2}^{*} / E_{1}^{*}} \rightarrow \mathcal{F}^{P} f^{-1} G
$$

Enfin, on considère la situation suivante : soient $f_{0}: Z_{1} \rightarrow Z_{2}$ un morphisme d'espace localement compact, $E_{1} \stackrel{\tau_{1}}{\rightarrow} Z_{1}$ et $E_{2} \stackrel{\tau_{2}}{\rightarrow} Z_{2}$ deux fibrés vectoriels, $g: E_{1} \rightarrow Z_{1} \underset{Z_{2}}{\times} E_{2}$ et $h:=f_{\tau} \circ h:$

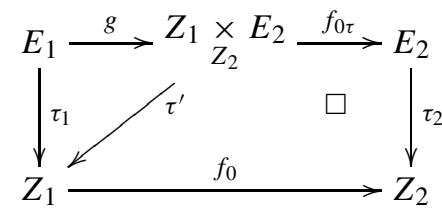

On pose $E_{2}^{\prime}:=Z_{1} \underset{Z_{2}}{\times} E_{2}$ et désignons par $E_{2}^{\prime *}$ son dual.

Définition 2.7. Soit $P \subset E_{2}^{\prime} \underset{Z_{1}}{\times} E_{2}^{* *}$ un ensemble de la forme (2.1). Pour tous objets $F \in D^{+}\left(E_{2}\right)$ et $G \in D^{+}\left(E_{1}\right)$, on définit

$$
\varphi \operatorname{hom}^{P}(G \rightarrow F):=\mathcal{F}^{P} \operatorname{RHom}\left(R g_{*} G, f_{0}^{-1} F\right) \in D^{+}\left(E_{2}^{*}\right) .
$$


Dans le cas où $Z_{1}=Z_{2}, f_{0}=\mathrm{id}, E:=E_{1}=E_{2}$, on pose pour tous $G, F \in D^{+}(E)$,

$$
\varphi \operatorname{hom}^{P}(G, F):=\varphi \operatorname{hom}^{P}(G \rightarrow F) \in D^{+}\left(E^{*}\right) .
$$

Remarquons que pour $G=\mathbb{C}_{E}$, on a évidement $\varphi \operatorname{hom}^{P}\left(\mathbb{C}_{E}, F\right)=\mathcal{F}^{P} F$.

\section{Opérateurs pseudo-différentiels non-locaux}

Maintenant, plaçons-nous au cas du complexe analytique: soient $X$ une variété complexe analytique, $T X$ le fibré tnagent au-dessus de $X$ et $T^{*} X$ le fibré cotangent, et $\tau: T X \rightarrow X$ et $\pi: T^{*} X \rightarrow X$ les projections. On désigne par $(x ; u),(y ; v)$, etc. les coordonnées sur $T X$ et par $(x ; \xi),(y ; \eta)$, etc. celles sur $T^{*} X$. Dans la suite, on emploiera l'isomorphisme suivant

$$
\psi:(x, y) \mapsto(x ; y-x): X \times X \stackrel{\sim}{\rightarrow} T X .
$$

On récrira la transformation de Fourier-Sato dans la presente situation: pour un compact $M_{0} \subset X$, on pose

$$
\begin{aligned}
H_{M_{0}}(\xi) & :=\sup _{u \in M_{0}} \operatorname{Re}\langle u, \xi\rangle, \\
I_{M_{0}}(\xi) & :=\inf _{u \in M_{0}} \operatorname{Re}\langle u, \xi\rangle
\end{aligned}
$$

où $\langle u, \xi\rangle:=\sum_{j=1}^{n} u_{j} \xi_{j}$ avec $u=\left(u_{1}, u_{2}, \ldots, u_{n}\right)$ et $\xi=\left(\xi_{1}, \xi_{2}, \ldots, \xi_{n}\right)$, que l'on désignera parfois par $u \cdot \xi$. Pour un fermé $\tau$-propre $M \subset T X$, on pose $M(x):=\tau^{-1}(M)$, pour tout $x \in X$, qui est un compact de $X$. Définissons

$$
P=P_{M}:=\left\{(x ; u ; \xi) \in T \underset{X}{\times} T^{*} X \mid \operatorname{Re}\langle u, \xi\rangle \leqslant H_{M(x)}(\xi)\right\} .
$$

Pour tout $F \in D^{b}(T X)$, on écrit

$$
\mathcal{F}^{M} F:=\mathcal{F}^{P_{M}} F=R \rho_{2 *} R \Gamma_{P_{M}} \rho_{1}^{-1} F \in D^{b}\left(T^{*} X\right) .
$$

Soit en suite, $f: Y \rightarrow X$ un morphisme de variété complexe. Pour tout fermé $M \subset Y \underset{X}{\times} T X \tau^{\prime}$-propre, $\tau^{\prime}: Y \underset{X}{\times} T X \rightarrow Y$ étant la projection, on définit $P=P_{M}$ comme (3.4) :

$$
P=P_{M}:=\left\{(x ; u ; \xi) \in Y \underset{X}{\times} T \underset{X}{\times} T^{*} X \mid \operatorname{Re}\langle u, \xi\rangle \leqslant H_{M(x)}(\xi)\right\} .
$$

Pour tous $G \in D^{b}(T Y)$ et $F \in D^{b}(T X)$, on désigne

$$
\varphi \operatorname{hom}^{M}(G \rightarrow F):=\varphi \operatorname{hom}^{P}(G \rightarrow F)
$$


où $D^{b}(\cdot)$ signifie la catégorie dérivée des complexes borné. Lorsque $Y=X, f=\operatorname{id}_{X}$, on écrit $\varphi \operatorname{hom}^{M}(G, F)=\varphi \operatorname{hom}^{M}(G \rightarrow F)$. Soient $\mathcal{O}_{X \times X}^{(0, n)}$ le faisceau des $(0, n)$-formes différentielles holomorphes sur $X \times X$ et $\omega_{T X / X}:=\tau^{!} \mathbb{C}_{X}$ et posons $\Omega_{X \times X / X}:=\mathcal{O}_{X \times X}^{(0, n)} \otimes \omega_{T X / X}$. Maintenant, on peut définir 'les opérateurs pseudodifférentiels non-locaux':

Définition 3.1. Pour tout fermé $\tau$-propre $M \subset T X$, on pose

$$
\mathcal{E}_{[M]}^{\mathbb{R}}:=\mathcal{F}^{M} R \psi_{*} \Omega_{X \times X / X} \in D^{b}\left(T^{*} X\right)
$$

et pour tout ouvert conique $V \subset T^{*} X$, on appelera $H^{0}\left(V, \mathcal{E}_{[M]}^{\mathbb{R}}\right)$ l'espace des opérateurs pseudo-différentiels non-locaux sur $V$.

Remarquons que $\mathcal{E}_{[M]}^{\mathbb{R}}$ n'est, en général, pas un faisceau sur $T^{*} X$, n'est défini que comme un objet de $D^{b}\left(T^{*} X\right)$. D'après la proposition 2.2, on a :

Proposition 3.2. Pour tout ouvert conique $V \subset T^{*} X$, si on pose

$$
\begin{aligned}
V^{M} & :=\psi^{-1}\left\{(x ; u) \in T X \mid \operatorname{Re}\langle u, \xi\rangle \leqslant H_{M(x)}(\xi)(\forall(x ; \xi) \in V)\right\} \\
& =\left\{(x, y) \in X \times X \mid \operatorname{Re}\langle y-x, \xi\rangle \leqslant H_{M(x)}(\xi)(\forall(x ; \xi) \in V)\right\},
\end{aligned}
$$

on $a$

$$
H^{0}\left(V, \mathcal{E}_{[M]}^{\mathbb{R}}\right) \simeq H_{V^{M}}^{n}\left(\pi(V) \times \mathbb{C}^{n}, \mathcal{O}_{X \times X}^{(0, n)}\right) \otimes \operatorname{or}_{T X / X}
$$

où $\operatorname{or}_{T X / X}$ est le faisceau d'orientation relative.

\section{Composition des opérateurs pseudo-différentiels non-locaux}

Pour définir la composition de deux opérateurs pseudo-différentiels non-locaux, nous commençons par un morphisme n'étant pas canonique

$$
\sigma:(x ; u) \mapsto x+u: T X \rightarrow X
$$

et le diagramme :

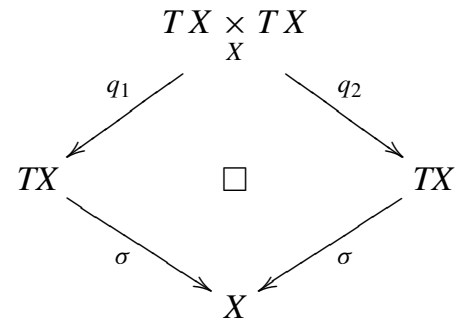


Et on définit aussi :

$$
q:(x ; u ; x+u ; v) \mapsto(x ; u+v): T \underset{X}{\times} T X \rightarrow T X,
$$

alors on a le diagramme :

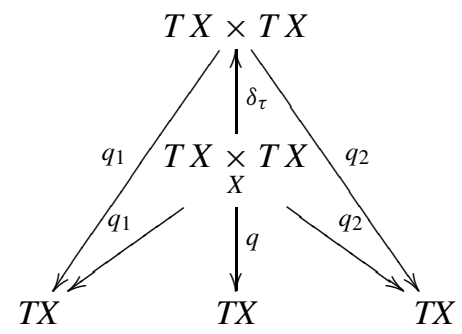

où $\delta_{\tau}: T X \times T X \hookrightarrow T X \times T X$ est l'inclusion canonique. Quant'à la dualité, on pose le fibre $E:=\mathbb{C}^{n}$ et soit $\phi:(x ; \xi) \mapsto \xi: T^{*} X \rightarrow E$. On définit le diagramme :

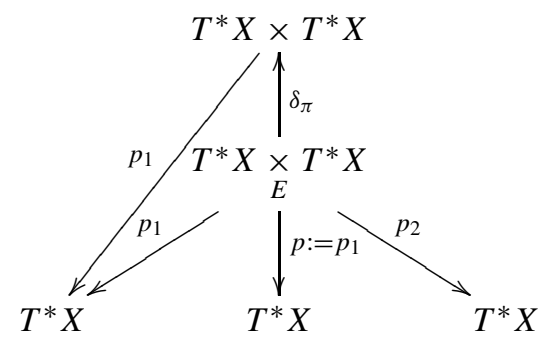

où $p_{1}, p_{2}$ sont des projections et $\delta_{\pi}$ l'inclusion canonique. On a l'inclusion :

$$
\delta_{\tau} \times \delta_{\pi}:(T X \underset{X}{\times} T X) \underset{X \times X}{\times}\left(T^{*} X \underset{E}{X} T^{*} X\right) \hookrightarrow T(X \times X) \underset{X \times X}{\times} T^{*}(X \times X),
$$

remarquons qu'en coordonné,

$$
(T X \underset{X}{\times} T X) \underset{X \times X}{\times}\left(T^{*} X \underset{E}{\times} T^{*} X\right)=\{((x ; u ; x+u ; v) ;(x ; \xi ; x+u ; \xi))\} .
$$

Définition 4.1. Soient $K, L, F, G \in D^{b}(T X)$. On pose

$$
\begin{gathered}
F \circ G:=R q_{!}\left(q_{1}^{-1} F \stackrel{\mathbb{L}}{\otimes} q_{2}^{-1} G\right), \\
K \circ \circ^{\prime} L:=R q_{*}\left(q_{1}^{-1} K \stackrel{\mathbb{L}}{\otimes} q_{2}^{-1} L\right) .
\end{gathered}
$$


On démontrera maintenant le théorème principal de cet article :

ThÉORÈme 4.2. On suppose que $M=X \times M_{0}, N=X \times N_{0}$ avec $M_{0}, N_{0} \subset \mathbb{C}^{n}$ deux compacts. Posons $M+N:=X \times\left(M_{0}+N_{0}\right)$. Pour tous $K, L, F, G \in D^{b}(T X)$, on a le morphisme canonique suivant que nous appelons la composition :

$$
R p_{!}\left(p_{1}^{-1} \varphi \operatorname{hom}^{M}(K, F) \stackrel{\mathbb{L}}{\otimes} p_{2}^{-1} \varphi \operatorname{hom}^{N}(L, G)\right) \rightarrow \varphi \operatorname{hom}^{M+N}\left(K \circ \circ^{\prime} L, F \circ G\right) .
$$

Démonstration. D’après la Proposition 2.3, on a

$$
\begin{aligned}
R p_{!}( & \left.p_{1}^{-1} \varphi \operatorname{hom}^{M}(K, F) \stackrel{\mathbb{L}}{\otimes} p_{2}^{-1} \varphi \operatorname{hom}^{N}(L, G)\right) \\
& =R p_{!} \delta_{\pi}^{-1}\left(\varphi \operatorname{hom}^{M}(K, F) \stackrel{\mathbb{L}}{\otimes} \varphi \operatorname{hom}^{N}(L, G)\right) \\
& =R p_{!} \delta_{\pi}^{-1}\left(\mathcal{F}^{M} \operatorname{RHom}(K, F) \stackrel{\mathbb{L}}{\otimes} \mathcal{F}^{N} \operatorname{RHom}(L, G)\right) \\
& \simeq R p_{!} \delta_{\pi}^{-1} \mathcal{F}^{M \times N}(\operatorname{RHom}(K, F) \stackrel{\mathbb{L}}{\otimes} \operatorname{RHom}(L, G)) .
\end{aligned}
$$

Posons

$$
\begin{aligned}
\tilde{P}:= & \left\{(x, y ; u, v ; \xi, \eta) \in T(X \times X) \underset{X \times X}{\times} T^{*}(X \times X) \mid \operatorname{Re}\langle u, \xi\rangle\right. \\
& \left.\leqslant H_{M_{0}}(\xi), \operatorname{Re}\langle v, \eta\rangle \leqslant H_{N_{0}}(\eta)\right\}
\end{aligned}
$$

et soient $T(X \times X) \stackrel{\tilde{\rho}_{1}}{\leftarrow} T(X \times X) \underset{X \times X}{\times} T^{*}(X \times X) \stackrel{\tilde{\rho}_{2}}{\rightarrow} T^{*}(X \times X)$ les projections.

Alors on a aussi facilement

$$
\begin{aligned}
& R p_{!} \delta_{\pi}^{-1} \mathcal{F}^{M \times N}(\operatorname{RHom}(K, F) \stackrel{\mathbb{L}}{\otimes} \operatorname{RHom}(L, G)) \\
& \quad \rightarrow R p_{!} \delta_{\pi}^{-1} \mathcal{F}^{M \times N} \operatorname{RHom}(K \underset{\mathbb{L}}{\otimes} L, F \underset{\mathbb{L}}{\mathbb{L}} G) \\
& \quad=R p_{!} \delta_{\pi}^{-1} R \tilde{\rho}_{2 *} R \Gamma_{\tilde{P}} \tilde{\rho}_{1}^{-1} \operatorname{RHom}(K \stackrel{\mathbb{L}}{\otimes} L, F \underset{\mathbb{L}}{\mathbb{L}} G) .
\end{aligned}
$$

Posons

$$
\begin{aligned}
\tilde{P}^{\prime}:= & \left(1 \times \delta_{\pi}\right)^{-1}(\tilde{P}) \\
= & \left\{(x, y ; u, v ; \xi) \in T(X \times X) \underset{X \times X}{\times}\left(T^{*} X \underset{X}{\times} T^{*} X\right) \mid \operatorname{Re}\langle u, \xi\rangle\right. \\
& \left.\leqslant H_{M_{0}}(\xi), \operatorname{Re}\langle v, \eta\rangle \leqslant H_{N_{0}}(\xi)\right\}
\end{aligned}
$$


et considérons

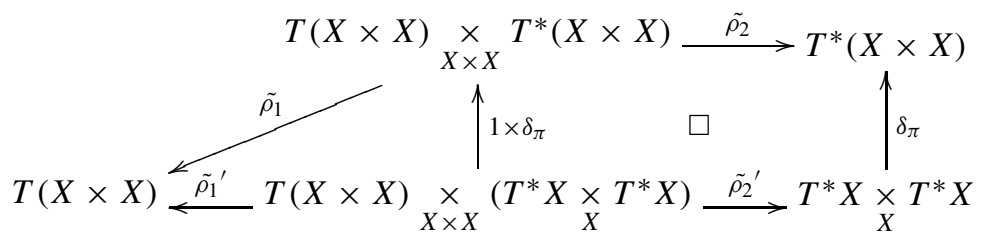

Les morphismes $\delta_{\pi}$ et $1 \times \delta_{\pi}$ étant injectifs, alors on a

$$
\begin{aligned}
R p ! \delta_{\pi}^{-1} R \tilde{\rho}_{2 *} R \Gamma_{\tilde{P}} \tilde{\rho}_{1}^{-1} & \rightarrow R p ! R \tilde{\rho}_{2 *}^{\prime}\left(1 \times \delta_{\pi}\right)^{-1} R \Gamma_{\tilde{P}} \tilde{\rho}_{1}^{-1} \\
& =R p ! R \tilde{\rho}_{2_{*}^{\prime}}^{\prime} R \Gamma_{\tilde{P}^{\prime}}\left(1 \times \delta_{\pi}\right)^{-1} \tilde{\rho}_{1}^{-1} \\
& =R p ! R \tilde{\rho}_{2_{*}^{\prime}}^{\prime} R \Gamma_{\tilde{P}^{\prime}} \tilde{\rho}_{1}^{\prime-1} .
\end{aligned}
$$

Posons encore

$$
\begin{aligned}
P^{\prime}:= & \left(\delta_{\tau} \times 1\right)^{-1}\left(\tilde{P}^{\prime}\right) \\
= & \{((x ; y-x),(y ; z-y),(x, y ; \xi)) \\
& \in(T X \times T X) \underset{X \times X}{\times}\left(T^{*} X \underset{X}{\times} T^{*} X\right) \mid \operatorname{Re}\langle y-x, \xi\rangle \\
& \left.\leqslant H_{M_{0}}(\xi), \underset{R e}{\operatorname{Re}}\langle z-y, \eta\rangle \leqslant H_{N_{0}}(\xi)\right\}
\end{aligned}
$$

et en considérant

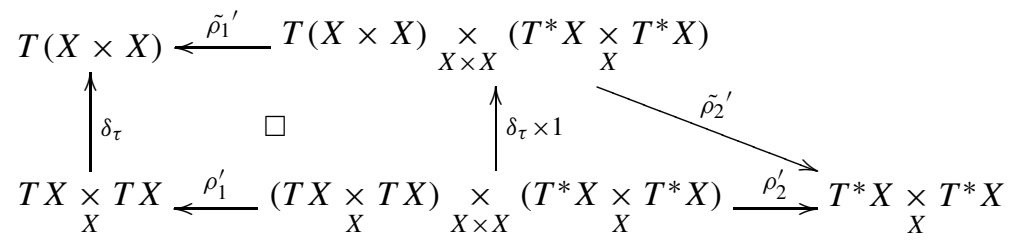

on a, $\delta_{\tau}$ étant injectif,

$$
\begin{aligned}
& R p ! R \tilde{\rho}_{2}^{\prime} R \Gamma_{\tilde{\rho}^{\prime}} \tilde{\rho}_{1}^{\prime-1} \rightarrow R p ! R \tilde{\rho}_{2}^{\prime} R \Gamma_{\tilde{\rho}^{\prime}} \tilde{\rho}_{1}^{\prime-1} R \delta_{\tau} \delta_{\tau}^{-1} \\
& =R p ! R \tilde{\rho}_{2}^{\prime} R \Gamma_{\tilde{P}^{\prime}} R\left(\delta_{\tau} \times 1\right)_{*} \rho_{1}{ }^{\prime-1} \delta_{\tau}^{-1} \\
& =R p ! R \tilde{\rho}_{2}^{\prime}{ }_{*}^{\prime} R\left(\delta_{\tau} \times 1\right)_{*} R \Gamma_{P^{\prime}} \rho_{1}{ }^{\prime-1} \delta_{\tau}^{-1} \\
& =R p ! R \rho_{2}{ }_{*} R \Gamma_{P^{\prime}} \rho_{1}{ }^{\prime-1} \delta_{\tau}^{-1} \text {. }
\end{aligned}
$$


Et posons

$$
\begin{aligned}
\hat{P}:= & (q \times 1)^{-1}\left(P^{\prime}\right) \\
= & \left\{((x ; z-x),(x, y ; \xi)) \in T \underset{X}{\times} \underset{X}{\times}\left(T^{*} X \underset{X}{\times} T^{*} X\right) \mid \operatorname{Re}\langle y-x, \xi\rangle\right. \\
& \left.\leqslant H_{M_{0}}(\xi), \operatorname{Re}\langle z-y, \eta\rangle \leqslant H_{N_{0}}(\xi)\right\}
\end{aligned}
$$

on a $P^{\prime}=(q \times 1)^{-1}(\hat{P})$ et compte tenu de

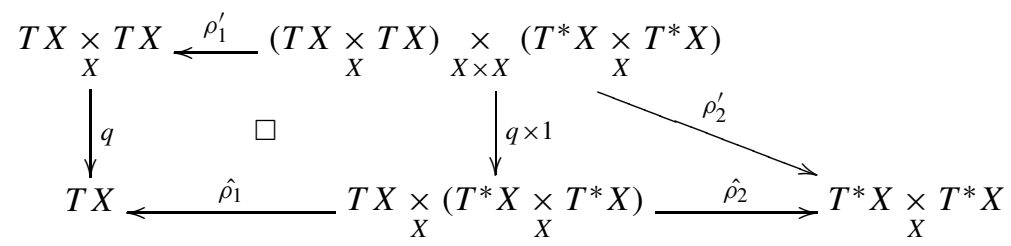

on a

$$
\begin{aligned}
R p_{!} R \rho_{2}{ }_{*} R \Gamma_{P^{\prime}} \rho_{1}{ }^{\prime-1} \delta_{\tau}^{-1} & \rightarrow R p_{!} R \hat{\rho_{2}} R(q \times 1)_{*} R \Gamma_{P^{\prime}} \rho_{1}{ }^{\prime-1} \delta_{\tau}^{-1} \\
& =R p_{!} R \hat{\rho}_{2 *} R \Gamma_{\hat{P}} R(q \times 1)_{*} \rho_{1}{ }^{\prime-1} \delta_{\tau}^{-1} \\
& =R p_{!} R \hat{\rho_{2 *}} R \Gamma_{\hat{P}}{\hat{\rho_{1}}}^{-1} R q_{!} \delta_{\tau}^{-1}
\end{aligned}
$$

Enfin posons

$$
\begin{aligned}
P:= & (1 \times p)(\hat{P}) \\
= & \left\{((x ; z-x),(x, ; \xi)) \in T X \underset{X}{\times} T^{*} X \mid \exists y \in X \text { t.q. } \operatorname{Re}\langle y-x, \xi\rangle\right. \\
& \left.\leqslant H_{M_{0}}(\xi), \operatorname{Re}\langle z-y, \eta\rangle \leqslant H_{N_{0}}(\xi)\right\}
\end{aligned}
$$

on a $\hat{P}=(1 \times p)^{-1}(P)$.

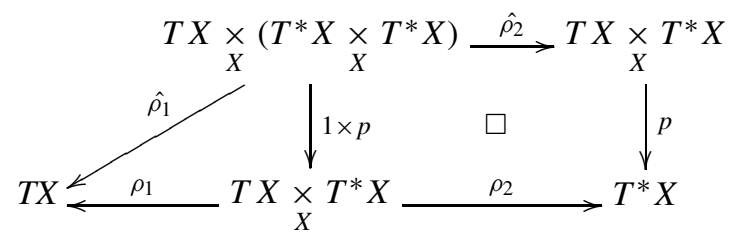


$1 \times p$ étant lisse, on a

$$
\begin{aligned}
R p_{!} R \hat{\rho_{2 *}} R \Gamma_{\hat{P}} \hat{\rho}_{1}^{-1} R q_{!} \delta_{\tau}^{-1} & \rightarrow R p_{*} R \hat{\rho}_{2 *} R \Gamma_{\hat{P}} \hat{\rho}_{1}^{-1} R q_{!} \delta_{\tau}^{-1} \\
& =R \rho_{2 *} R(1 \times p)_{*} R \Gamma_{\hat{P}} \hat{\rho}_{1}^{-1} R q_{!} \delta_{\tau}^{-1} \\
& =R \rho_{2 *} R \Gamma_{P} R(1 \times p)_{*} \hat{\rho}_{1}^{-1} R q_{!} \delta_{\tau}^{-1} \\
& =R \rho_{2 *} R \Gamma_{P} R(1 \times p)_{*}(1 \times p)^{-1} \rho_{1}^{-1} R q_{!} \delta_{\tau}^{-1} \\
& =R \rho_{2 *} R \Gamma_{P} \rho_{1}^{-1} R q_{!} \delta_{\tau}^{-1} .
\end{aligned}
$$

Bien-entendu, on a

$$
\begin{aligned}
& P=\left\{((x ; z-x),(x, ; \xi)) \in T \underset{X}{X} \underset{X}{\times} T^{*} X \mid \exists y \in X \text { t.q. } \operatorname{Re}\langle y-x, \xi\rangle\right. \\
& \left.\quad \leqslant H_{M_{0}}(\xi), \operatorname{Re}\langle z-y, \eta\rangle \leqslant H_{N_{0}}(\xi)\right\} \\
& \subset\left\{((x ; z-x),(x, ; \xi)) \in T \underset{X}{X} \underset{X}{\times} T^{*} X \mid \operatorname{Re}\langle z-x, \xi\rangle \leqslant H_{M_{0}+N_{0}}(\xi)\right\}=P_{M+N}
\end{aligned}
$$

et donc on a

$$
\begin{aligned}
R \rho_{2 *} R \Gamma_{P} \rho_{1}^{-1} R q_{!} \delta_{\tau}^{-1} & \rightarrow R \rho_{2 *} R \Gamma_{P_{M+N} \rho_{1}^{-1} R q_{!} \delta_{\tau}^{-1}} \\
& =\mathcal{F}^{M+N} R q_{!} \delta_{\tau}^{-1} .
\end{aligned}
$$

D'où, compte tenu de (2.6.26) et (2.6.25) de [2], (4.8) donne

$$
\begin{aligned}
R p_{!} & \delta_{\pi}^{-1} \mathcal{F}^{M \times N}(\operatorname{RHom}(K, F) \stackrel{\mathbb{L}}{\otimes} \operatorname{RHom}(L, G)) \\
& \rightarrow R p_{!} \delta_{\pi}^{-1} R \tilde{\rho}_{2 *} R \Gamma_{\tilde{P}} \tilde{\rho}_{1}^{-1} \operatorname{RHom}(K \stackrel{\mathbb{L}}{\otimes} L, F \underset{\mathbb{L}}{\otimes} G) \\
& \rightarrow \mathcal{F}^{M+N} R q_{!} \delta_{\tau}^{-1} \operatorname{RHom}(K \stackrel{\mathbb{L}}{\otimes} L, F \stackrel{\mathbb{L}}{\otimes} G) \\
& \rightarrow \mathcal{F}^{M+N} \operatorname{RHom}\left(R q_{*} \delta_{\tau}^{-1}(K \stackrel{\mathbb{L}}{\otimes} L), R q_{!} \delta_{\tau}^{-1}(F \mathbb{\mathbb { L }} G)\right) \\
& =\varphi \operatorname{hom}^{M+N}\left(K \circ^{\prime} L, F \circ G\right) .
\end{aligned}
$$

Comme corollaire du théorème, on a la composition de deux opérateurs pseudodifférentiels non-locaux, qui coïncides à celle défini dans la proposition 3.5 de [1] :

THÉORÈmE 4.3. Soient $M_{0}, N_{0} \subset \mathbb{C}^{n}$ deux compacts et on pose $M:=X \times M_{0}, N:=$ $X \times N_{0}$. Alors, on a le morphisme de compositions suivantes :

$$
R p_{!}\left(p_{1}^{-1} \mathcal{E}_{[M]}^{\mathbb{R}} \stackrel{\mathbb{L}}{\otimes} p_{2}^{-1} \mathcal{E}_{[N]}^{\mathbb{R}}\right) \rightarrow \mathcal{E}_{[M+N]}^{\mathbb{R}}
$$


Démonstration. Dans le théorème 4.2, on pose $F=G:=R \psi_{*} \Omega_{X \times X / X}$ et $K=L:=$ $\mathbb{C}_{T X}$. Alors en remarquons $\mathbb{C}_{T X}$ o $^{\prime} \mathbb{C}_{T X} \simeq \mathbb{C}_{T X}$, on a

$$
\begin{aligned}
R & p_{!}\left(p_{1}^{-1} \mathcal{E}_{[M]}^{\mathbb{R}} \stackrel{\mathbb{L}}{\otimes} p_{2}^{-1} \mathcal{E}_{[N]}^{\mathbb{R}}\right) \\
\quad & R p_{!}\left(p_{1}^{-1} \varphi \operatorname{hom}^{M}\left(\mathbb{C}_{T X}, R \psi_{*} \Omega_{X \times X / X}\right) \stackrel{\mathbb{L}}{\otimes} p_{2}^{-1} \varphi \operatorname{hom}^{N}\left(\mathbb{C}_{T X}, R \psi_{*} \Omega_{X \times X / X}\right)\right) \\
& \rightarrow \varphi \operatorname{hom}^{M+N}\left(\mathbb{C}_{T X}, R \psi_{*} \Omega_{X \times X / X} \circ R \psi_{*} \Omega_{X \times X / X}\right) .
\end{aligned}
$$

D'après e.g. le théorème 11.1.4 de [2], on a $R \psi_{*} \Omega_{X \times X / X} \circ R \psi_{*} \Omega_{X \times X / X} \stackrel{\int_{q}}{\rightarrow}$ $R \psi_{*} \Omega_{X \times X / X}$ et d'où l'on a

$$
\begin{aligned}
& \varphi \operatorname{hom}^{M+N}\left(\mathbb{C}_{T X}, R \psi_{*} \Omega_{X \times X / X} \circ R \psi_{*} \Omega_{X \times X / X}\right) \\
& \quad \rightarrow \varphi \operatorname{hom}^{M+N}\left(\mathbb{C}_{T X}, R \psi_{*} \Omega_{X \times X / X}\right) \\
& =\mathcal{E}_{[M+N]}^{\mathbb{R}} .
\end{aligned}
$$

\section{RÉFÉRENCES}

[1] R. Ishimura. Non-local pseudo-differential operators. J. Math. Pures Appl. 81 (2002), 1241-1276.

[2] M. Kashiwara et P. Schapira. Sheaves on Manifolds (Grundlehren der Mathematischen Wissenschaften, 292). Springer, New York, 1990.

[3] M. Sato, T. Kawai et M. Kashiwara. Microfunctions and Pseudo-differential Equations (Lecture Notes in Mathematics, 287). Springer, Berlin, 1973, pp. 264-529.

Ryuichi Ishimura

Department of Mathematics and Informatics Faculty of Science

Chiba University

Yayoicho Chiba 263-8522

Japan

(E-mail: ishimura@math.s.chiba-u.ac.jp) 